\title{
Applications of Nonrelativistic Effective Field Theories to quarkonium systems with a small radius
}

\author{
Nora Brambilla ${ }^{\mathrm{a}}$ \\ aINFN and Dipartimento di Fisica dell'universita' di Milano, \\ Via Celoria 16, 20133 Milan, Italy
}

We review some predictions of nonrelativistic effective field theories for heavy quark-antiquark systems with a small typical radius, $r<1 / \Lambda_{\mathrm{QCD}}$.

\section{INTRODUCTION}

We are interested here in bound states composed only by heavy quarks (and gluons). In such cases, at least for the lowest states, the characteristic radius $r$ ( $r$ being the $q \bar{q}$ distance) of the system is small, typically too small to probe the confinement effects, which arise at a scale $1 / \Lambda_{\mathrm{QCD}} \gg r$. In the following I will call such systems 'Coulombic' or 'quasi-Coulombic'. These situations are particularly interesting not only because they have phenomenological relevance, but especially because they allow us to understand much more about QCD. In such cases both the mass scale $m$ and the soft scale $1 / r$ are much bigger than $\Lambda_{\mathrm{QCD}}$ and thus still sit in the perturbative regime. Non-perturbative corrections exist but are not expected to be dominant.

As it is apparent from the spectra, heavy quarkonia are non-relativistic systems. Thus, they may be described in first approximation using a Schrödinger equation with a potential interaction. This amounts to saying that the heavy quark bound state is characterized by three energy scales, hierarchically ordered by the quark velocity $v \ll 1$ : the quark mass $m$ (hard scale), the momentum $m v \simeq 1 / r$ (soft scale $(\mathrm{S})$ ), and the binding energy $m v^{2}$ (ultrasoft scale (US)). In the Coulombic or quasi-Coulombic situation it is $v \sim \alpha_{\mathrm{s}}$.

To address the multiscale dynamics of the heavy quark bound state, the concept of effective field theory turns out to be not only helpful but actually necessary. QCD effective field theories (EFT) with less and less degrees of freedom, can be obtained by systematically integrating out the scales above the energy we aim to describe. This procedure leads ultimately to a field theory derived quantum mechanical description of these systems. The corresponding EFT is called pNRQCD [1]. Here, all the dynamical regimes are organized in a systematic expansion in the ratio of the different scales which is eventually an expansion in $v$.

In practice, by integrating out the hard scale NRQCD is obtained from QCD. After integrating out the soft scale in NRQCD, pNRQCD is obtained. The Lagrangian of pNRQCD is organized in powers of $1 / m$ and $\mathbf{r}$ (multipole expansion). The matching is done by comparing appropriate off-shell amplitudes in NRQCD and in pNRQCD, order by order in $1 / m, \alpha_{s}$ and order by order in the multipole expansion. The matching coefficients are non-analytic functions of $\mathbf{r}$ and have typically the following structure: $V \simeq \mathcal{V}\left(\mathbf{r}, \mathbf{p}, \mathbf{S}_{\mathbf{1}}, \mathbf{S}_{2}\right)\left(A \ln m r+B \ln \mu^{\prime} r+C\right)$.

\section{2. pNRQCD for $m v \gg \Lambda_{\mathrm{QCD}}$}

At the scale of the matching $\mu^{\prime}\left(m v \gg \mu^{\prime} \gg\right.$ $\left.m v^{2}, \Lambda_{\mathrm{QCD}}\right)$ we have still quarks and gluons. We denote by $\mathbf{R} \equiv\left(\mathbf{x}_{1}+\mathbf{x}_{2}\right) / 2$ the center-of-mass of the $q \bar{q}$ system and by $\mathbf{r} \equiv \mathbf{x}_{\mathbf{1}}-\mathbf{x}_{\mathbf{2}}$ the relative distance. The effective degrees of freedom are: $q \bar{q}$ states (that can be decomposed into a singlet $S(\mathbf{R}, \mathbf{r}, t)$ and an octet $O(\mathbf{R}, \mathbf{r}, t)$ under 
Table 1

Summary of the different kinematical situations.

\begin{tabular}{llll}
\hline$m v$ & $m v^{2}$ & potential & ultrasoft corrections \\
\hline$\gg \Lambda_{\mathrm{QCD}}$ & $\gg \Lambda_{\mathrm{QCD}}$ & perturbative & local condensates \\
$\gg \Lambda_{\mathrm{QCD}}$ & $\sim \Lambda_{\mathrm{QCD}}$ & perturbative & non-local condensates \\
$\gg \Lambda_{\mathrm{QCD}}$ & $\ll \Lambda_{\mathrm{QCD}}$ & perturbative + & No US (if light quarks \\
& & short-range nonpert. & are not considered) \\
\hline
\end{tabular}

color transformations) with energy of the order of the next relevant scale, $O\left(\Lambda_{Q C D}, m v^{2}\right)$, and momentum ${ }^{1} \mathbf{p}$ of order $O(m v)$, plus ultrasoft gluons $A_{\mu}(\mathbf{R}, t)$ with energy and momentum of order $O\left(\Lambda_{\mathrm{QCD}}, m v^{2}\right)$. Notice that all the gluon fields are multipole expanded. The Lagrangian is then an expansion in the small quantities $p / m, 1 / r m$ and in $O\left(\Lambda_{\mathrm{QCD}}, m v^{2}\right) \times r$. The pNRQCD Lagrangian is given in this case at the leading order in the multipole expansion by[1]:

$$
\begin{aligned}
& \mathcal{L}=\operatorname{Tr}\left\{\mathrm{S}^{\dagger}\left(i \partial_{0}-\frac{\mathbf{p}^{2}}{m}-V_{s}(r)-\sum_{n=1} \frac{V_{s}^{(n)}}{m^{n}}\right) \mathrm{S}\right. \\
& \left.+\mathrm{O}^{\dagger}\left(i D_{0}-\frac{\mathbf{p}^{2}}{m}-V_{o}(r)-\sum_{n=1} \frac{V_{o}^{(n)}}{m^{n}}\right) \mathrm{O}\right\} \\
& +g V_{A}(r) \operatorname{Tr}\left\{\mathrm{O}^{\dagger} \mathbf{r} \cdot \mathbf{E} \mathrm{S}+\mathrm{S}^{\dagger} \mathbf{r} \cdot \mathbf{E} \mathrm{O}\right\} \\
& +g \frac{V_{B}(r)}{2} \operatorname{Tr}\left\{\mathrm{O}^{\dagger} \mathbf{r} \cdot \mathbf{E} \mathrm{O}+\mathrm{O}^{\dagger} \mathrm{Or} \cdot \mathbf{E}\right\}-\frac{1}{4} F_{\mu \nu}^{a} F^{\mu \nu a} .
\end{aligned}
$$

All the gauge fields in Eq. (1) are evaluated in $\mathbf{R}$ and $t$. In particular $\mathbf{E} \equiv \mathbf{E}(\mathbf{R}, t)$ and $i D_{0} \mathrm{O} \equiv$ $i \partial_{0} \mathrm{O}-g\left[A_{0}(\mathbf{R}, t), \mathrm{O}\right]$. The quantities denoted by $V$ are the matching coefficients.

We call $V_{s}$ and $V_{o}$ the singlet and octet static matching potentials respectively. At the leading order in the multipole expansion, the singlet sector of the Lagrangian gives rise to equations of motion of the Schrödinger type. The two last lines of (1) contain (apart from the Yang-Mills Lagrangian) retardation (or non-potential) effects that start at the NLO in the multipole expansion. At this order the non-potential effects come from the singlet-octet and octet-octet interactions mediated by a ultrasoft chromoelectric field. Recalling that $\mathbf{r} \sim 1 / m v$ and that the operators count

\footnotetext{
${ }^{1}$ Although, for simplicity, we describe the matching between NRQCD and pNRQCD as integrating out the soft scale, the relative momentum $\mathbf{p}$ of the quarks is still soft.
}

like the next relevant scale, $O\left(m v^{2}, \Lambda_{\mathrm{QCD}}\right)$, to the power of the dimension, it follows that each term in the pNRQCD Lagrangian has a definite power counting. This feature makes $\mathcal{L}_{\mathrm{pNRQCD}}$ a suitable tool for bound state calculations: being interested in knowing the energy levels up to some power $v^{n}$, we just need to evaluate the contributions of this size from the Lagrangian. From the power counting e.g., it follows that the interaction of quarks with ultrasoft gluons (nonpotential or retardation effect) is suppressed in the Lagrangian by $v$ with respect to the LO ( by $g v$ if $m v^{2} \gg \Lambda_{\mathrm{QCD}}$ ).

In particular, pNRQCD provides us with the way of obtaining the matching potentials via the matching procedure at any order of the perturbative expansion[1]. In the EFT language the potential is defined upon the integration of all the scales up to the ultrasoft scale $m v^{2}$. From the matching to NRQCD in the situation $\Lambda_{\mathrm{QCD}} \ll$ $m v$ we can easily obtain the matching potential $V_{s}$ at $\mathrm{N}^{3} \mathrm{LL}[1]$

$$
\begin{aligned}
& V_{s}(r) \equiv-C_{F} \frac{\alpha_{V}\left(r, \mu^{\prime}\right)}{r}, \\
& \alpha_{V}(r, \mu)=\alpha_{\mathrm{s}}(r)\left\{1+\left(a_{1}+2 \gamma_{E} \beta_{0}\right) \frac{\alpha_{\mathrm{s}}(r)}{4 \pi}\right. \\
& +\frac{\alpha_{\mathrm{S}}^{2}(r)}{16 \pi^{2}}\left[\gamma_{E}\left(4 a_{1} \beta_{0}+2 \beta_{1}\right)+\left(\frac{\pi^{2}}{3}+4 \gamma_{E}^{2}\right) \beta_{0}^{2}\right. \\
& \left.\left.+a_{2}\right]+\frac{C_{A}^{3}}{12} \frac{\alpha_{\mathrm{s}}^{3}(r)}{\pi} \ln r \mu^{\prime}\right\},
\end{aligned}
$$

where $\beta_{n}$ are the coefficients of the beta function ( $\alpha_{\mathrm{s}}$ is in the $\overline{\mathrm{MS}}$ scheme), and $a_{1}$ and $a_{2}$ were given in [2]. The Coulomb potential turns out to be sensitive to the ultrasoft scale but infrared finite. The same happens with the other potentials (like $V_{o}$ or the potentials that bear corrections in $1 / m^{n}$ ) that can equally be calculated via the matching procedure. The $\mu^{\prime}$ scale dependence in 
the potential is cancelled for any physical process by the contribution of the ultrasoft gluons that are cutoff at the scale $\mu^{\prime}$.

Then, there are two main situations [1], cf. Table 1 . If $m v \gg m v^{2} \gtrsim \Lambda_{\mathrm{QCD}}$, the system is described up to order $\alpha_{\mathrm{s}}^{4}$ by a potential which is entirely accessible to perturbative QCD. Non-potential effects start at order $\alpha_{\mathrm{s}}^{5} \ln \mu^{\prime}[3,4]$. We call Coulombic this kind of system. Non-perturbative effects are of nonpotential type and can be encoded into local (à la Voloshin-Leutwyler) or non-local condensates: they are suppressed by powers of $\Lambda_{\mathrm{QCD}} / m v^{2}$ and $\Lambda_{\mathrm{QCD}} / m v$ respectively. If $m v \gg \Lambda_{\mathrm{QCD}} \gg m v^{2}$, the scale $m v$ can be still integrated out perturbatively, giving rise to the Coulomb-type potential (2). Non-perturbative contributions to the potential arise when integrating out the scale $\Lambda_{\mathrm{QCD}}$ [1]. We call quasi-Coulombic the systems where the non-perturbative piece of the potential can be considered small with respect to the Coulombic one and treated as a perturbation. Some levels of $t \bar{t}$, the lowest level of $b \bar{b}$ may be considered Coulombic systems, while the $J / \psi$, the $\eta_{c}$ and the short-range hybrids may be considered quasiCoulombic. The $B_{c}$ may be in a boundary situation. As it is typical in an effective theory, only the actual calculation may confirm if the initial assumption about the physical system was appropriate.

For all these systems it is relevant to obtain a determination of the energy levels as accurate as possible in perturbation theory. In recent years our knowledge of heavy quarkonia in the framework of perturbative QCD has developed significantly. On one hand computations of new higher-order corrections to various physical quantities appeared e.g. $[3,4,6]$. On the other hand the discovery of the mechanism of the renormalon cancellation in the quarkonium spectrum led to a drastic improvement of the convergence of the perturbative expansion of the energy levels. As important applications up to date, the theory enabled precise determinations of the $\overline{\mathrm{MS}}$-mass of the bottom quark and of the top quark from (mainly) the energy spectra of the lowest-lying states. The main uncertainty comes, in the bottomonium case, from the (essentially) unknown non-perturbative contributions. These are generally claimed to be around 100 $\mathrm{MeV}$ and ultimately set the precision of the prediction. Within pNRQCD a complete and systematic parametrization of perturbative and nonperturbative effects of the heavy quarkonium interaction can be performed (cf. Table 1 and [1]). The parametrization depends on the mutual relation between the scale of non-perturbative physics, $\Lambda_{\mathrm{QCD}}$, and the other dynamical scales in the specific heavy quarkonium system under study. A way to determine it and the size and nature of the non-perturbative contributions, consists in establishing to which extent perturbative QCD can consistently and successfully describe the system. This is the problem we have addressed in[5], which investigates the range of validity of perturbative QCD on the heavy quarkonium spectroscopy and consequently extracts upper bounds to the non-perturbative contributions by comparing the perturbative predictions, at the current best accuracy, with the experimental data. One of the main problems in having a consistent (i.e. convergent) perturbative expansion for the quarkonium energy levels has been for a long time its bad behaviour when expressed in terms of the pole mass. The reason has been understood in the renormalon language[7]: the pole mass and the QCD static potential, respectively, contain renormalon contributions of order $\Lambda_{\mathrm{QCD}}$, which get cancelled in the total energy of a color singlet quark-antiquark system. The solution then consists in making explicit this cancellation by substituting in the energy expansion a short-range mass (free from infrared ambiguities) for the pole mass. In order to make explicit the renormalon cancellation we will express the quarkonium energies in terms of the $\overline{\mathrm{MS}}$ masses and rearrange the perturbative series in the socalled $\varepsilon$ expansion [8]. This will be the key ingredient of our analysis. We note that according to a formal argument, the residual uncertainty of the perturbative expansion due to the next-toleading renormalon contribution is estimated to be $\Lambda_{\mathrm{QCD}} \times\left(a_{X} \Lambda_{\mathrm{QCD}}\right)^{2}$ for a bound-state $X$ of size $a_{X}[1,5]$. Provided this argument applies, in principle the predictions of perturbative QCD can be 
Table 2

The quantities $E_{X}^{(j)}$ are the order $\varepsilon^{j}$ contributions to the spectrum [5,8]. The c-and b-quark msbar masses are fixed on the experimental values of the $J / \psi$ and $\Upsilon(1 S)$ masses. The uncertainties in the third and fourth columns refer to the uncertainties in $\alpha_{\mathrm{s}}^{(5)}\left(M_{Z}\right)$ only. All the other data refer to $\alpha_{\mathrm{s}}^{(5)}\left(M_{Z}\right)=0.1181$ and to the msbar quark masses fixed on the central values.

\begin{tabular}{lllllllll}
\hline State $X$ & $E_{X}^{\exp }$ & $E_{X}$ & $E_{X}^{\exp }-E_{X}$ & $E_{X}^{(1)}$ & $E_{X}^{(2)}$ & $E_{X}^{(3)}$ & $\mu_{X}^{A}$ & $\alpha_{\mathrm{s}}^{(3)}\left(\mu_{X}^{A}\right)$ \\
\hline$\Upsilon\left(1^{3} S_{1}\right)$ & 9.460 & 9.460 & 0 & 0.866 & 0.208 & 0.006 & 2.14 & 0.286 \\
$\Upsilon\left(1^{3} P_{0}\right)$ & 9.860 & $9.995_{-62}^{+75}$ & $-0.135_{+62}^{-75}$ & 1.534 & 0.101 & -0.021 & 1.08 & 0.459 \\
$\Upsilon\left(1^{3} P_{1}\right)$ & 9.893 & $10.004_{-63}^{+78}$ & $-0.111_{+63}^{-78}$ & 1.564 & 0.081 & -0.022 & 1.05 & 0.468 \\
$\Upsilon\left(1^{3} P_{2}\right)$ & 9.913 & $10.012_{-65}^{+81}$ & $-0.099_{+65}^{-81}$ & 1.591 & 0.063 & -0.022 & 1.034 & 0.477 \\
$\Upsilon\left(2^{3} S_{1}\right)$ & 10.023 & $10.084_{-75}^{+93}$ & $-0.061_{+93}^{-93}$ & 1.618 & 0.096 & -0.010 & 1.02 & 0.486 \\
$\Upsilon\left(2^{3} P_{0}\right)$ & 10.232 & $10.548_{-151}^{+196}$ & $-0.316_{+151}^{-196}$ & 2.421 & -0.356 & 0.102 & 0.778 & 0.710 \\
$\Upsilon\left(2^{3} P_{1}\right)$ & 10.255 & $10.564_{-153}^{+200}$ & $-0.309_{+153}^{-150}$ & 2.472 & -0.404 & 0.116 & 0.770 & 0.726 \\
$\Upsilon\left(2^{3} P_{2}\right)$ & 10.268 & $10.578_{-155}^{+203}$ & $-0.310_{+155}^{-203}$ & 2.518 & -0.449 & 0.129 & 0.762 & 0.740 \\
$\Upsilon\left(3^{3} S_{1}\right)$ & 10.355 & $10.645_{-168}^{+218}$ & $-0.290_{+168}^{-218}$ & 2.472 & -0.348 & 0.140 & 0.770 & 0.726 \\
$\Upsilon\left(4^{3} S_{1}\right)$ & 10.580 & $*$ & $*$ & $*$ & $*$ & $*$ & $*$ & $*$ \\
$B_{c}\left(1^{1} S_{0}\right)$ & $6.4 \pm 0.4$ & $6.307_{-2}^{+4}$ & $0.1 \pm 0.4$ & 0.675 & 0.188 & 0.017 & 1.62 & 0.334 \\
\hline
\end{tabular}

made precise down to this accuracy by sufficiently increasing the orders of the perturbative expansion. The complete analysis of the consistency between the whole level structure of perturbative QCD and the experimental data has been done in [5]. The major results of that analysis have been as follows (see Table 2). (1) Once the cancellation of the leading renormalon has been incorporated, the perturbative series turns out to be convergent and to reproduce reasonably well the gross structure of the bottomonium spectrum at least up to some of the $n=3$ levels. (2) The constraints on non-perturbative and higher-order contributions to the bottomonium spectrum, set by the comparison of the calculation with the experimental data, indicate that these are smaller than usually believed. Moreover, the perturbative predictions of the bottomonium spectrum up to $n=3$ appears to be in agreement with the experimental data (within the next-to-leading renormalon uncertainties and taken into account the error with which we know $\alpha_{s}$ ).

\section{REFERENCES}

1. N. Brambilla, A. Pineda, J. Soto and A. Vairo, Nucl. Phys. B 566, 275 (2000) N. Brambilla, A. Pineda, J. Soto and
A. Vairo, Phys. Rev. D 60, 091502 (1999) A. Pineda and J. Soto, Nucl. Phys. Proc. Suppl. 64, 428 (1998)

2. Y. Schroder, Phys. Lett. B 447, 321 (1999) M. Peter, Nucl. Phys. B 501, 471 (1997)

3. N. Brambilla, A. Pineda, J. Soto and A. Vairo, Phys. Lett. B 470, 215 (1999)

4. B. A. Kniehl, A. A. Penin, V. A. Smirnov and M. Steinhauser, Nucl. Phys. B 635, 357 (2002)

5. N. Brambilla, Y. Sumino and A. Vairo, Phys. Rev. D 65, 034001 (2002) N. Brambilla, Y. Sumino and A. Vairo, Phys. Lett. B 513, 381 (2001) S. Recksiegel and Y. Sumino, S. Recksiegel and Y. Sumino, arXiv:hepph/0207005. N. Brambilla and A. Vairo, Phys. Rev. D 62, 094019 (2000) [arXiv:hepph/0002075].

6. A. A. Penin and M. Steinhauser, Phys. Lett. B 538, 335 (2002) A. Pineda, JHEP 0106, 022 (2001) J. H. Kuhn and M. Steinhauser, Nucl. Phys. B 619, 588 (2001) [Erratum-ibid. B 640, 415 (2002)] A. H. Hoang,

7. A. H. Hoang, M. C. Smith, T. Stelzer and S. Willenbrock, Phys. Rev. D 59, 114014 (1999)

8. A. H. Hoang, Z. Ligeti and A. V. Manohar, Phys. Rev. Lett. 82, 277 (1999) 